\title{
Philosophiques
}

\section{Ontologie, esthétique et oeuvre d'art littéraire}

\section{Naïm Kattan}

Volume 5, numéro 2, octobre 1978

URI : https://id.erudit.org/iderudit/203100ar

DOI : https://doi.org/10.7202/203100ar

Aller au sommaire du numéro

Éditeur(s)

Société de philosophie du Québec

ISSN

0316-2923 (imprimé)

1492-1391 (numérique)

Découvrir la revue

Citer cet article

Kattan, N. (1978). Ontologie, esthétique et oeuvre d'art littéraire. Philosophiques, 5(2), 261-269. https://doi.org/10.7202/203100ar d'utilisation que vous pouvez consulter en ligne.

https://apropos.erudit.org/fr/usagers/politique-dutilisation/ 


\title{
ONTOLOGIE, ESTHÉTIQUE et OEUVRE D'ART LITTÉRAIRE
}

\author{
par Naïm Kattan
}

Pour que l'œuvre d'art ne soit pas problématique, il suffirait pour l'artiste de prétendre que son intention était de créer, de faire surgir du néant un produit, un objet incandescent offert au déchiffrement et à la lecture et qui garderait, à l'abri du temps et de l'espace, son mystère. L'artiste se contenterait de son acte et son produit, bouteille jetée à la mer, serait à la fois la question et la réponse. L'acte accompli, l'objet détiendrait une autonomie et le hasard ferait qu'il meure ou qu'il vive selon qu'il y aurait ou non rencontre avec un lecteur ou un spectateur. D'emblée, l'artiste accepterait ainsi le risque non seulement du hasard mais de la futilité, voire de l'inexistence. Une telle prétention serait donc pire qu'un rêve, qu'une vanité : une illusion, un leurre, et surtout un piège que dresse une bonne conscience à l'affût de confirmation.

L'ouvre d'art affirme, doit affirmer son autonomie qui n'est pas une donnée mais une liberté arrachée aux contingences, aux rapports avec le lieu, le temps et un public.

L'artiste fournit un travail mais l'impulsion qui le pousse et le conduit n'est point uniquement de produire un objet. Il lutte pour son existence, mais il s'agit pour lui de l'essentiel qu'aucune satisfaction immédiate ne peut combler, et si chez lui la voix du désir se taisait, s'il parvenait au consentement, la paix qu'il atteindrait serait celle de la résignation, de la mort, autrement dit du silence.

Le point de départ pour l'artiste est une appréhension du réel et une inaptitude, une difficulté d'établir avec ce réel un lien persistant, continu, sinon cohérent. C'est l'incohérence du monde, du réel, qui reflète, tel un miroir, l'incohérence d'une 
saisie possible et constitue pour l'artiste l'impulsion première de la tenter. Saisir le réel pour affirmer sa place dans le monde, le refléter, le dire pour affirmer une vie qui intègre le réel et s'y oppose, arrache son mystère et s'y perd.

Pour l'artiste, l'œuvre ne peut pas être seulement liée au réel, à la causalité et à la finitude, mais aussi à la vie, être et présence, dans l'expression souveraine de sa quotidienneté. L'instant est plénitude et l'artiste, comme tout homme, en ressent la mesure, mais connaît avec cette conscience de l'instant, l'éphémère, son passage, fulgurance et mort, éclat et immobilité. Autrement dit, il affronte le manque parce qu'il a ressenti la plénitude et c'est alors qu'il se met à la poursuite de l'éphémère, cherchant à le fixer, à le situer dans une continuité et tout au moins à exprimer, comme un malheur, le passage, l'absence qui suit tout surgissement, le silence qui couvre de son épaisseur l'éclatement de la parole et de l'image. Je ne crois pas que l'artiste accepte que son entreprise ne soit que conjuration d'un malheur, compensation d'un manque.

La plénitude intégrée dans une conscience apparaît non seulement comme éphémère mais comme inscription dans le temps. Elle ne se suffit pas à elle-même. Elle se veut durée, d'où la tentative de l'artişte de poursuivre l'instant, par surcroît de vie, trop plein d'épuisement du vécu dans son mouvement, et l'auvre devient alors tentative non pas de fixation, même s'il y aboutit, mais continuité de mouvement.

Toute œuvre serait alors la poursuite non d'un acte manqué mais d'une action continue, d'une entreprise inachevée. Les limites de l'existence humaine découlent des limites du corps, et Antonin Artaud s'est insurgé contre elles dans un cri tragique et impuissant, mais aussi dans la limite du réel tel qu'il est possible de le vivre. Si le vécu est par nature continuité mais aussi inachèvement, il ne suffit plus de comprendre, d'analyser, de mettre de l'ordre dans l'anarchie des faits et des actes pour affronter l'incohérence et y mettre un terme. Il importe de reconstruire le réel, de le refaire, de l'établir, de le fonder dans l'imaginaire. Pour les surréalistes, cet imaginaire n'était pas uniquement un soubassement, un arrière-plan, c'était le réel lui-même dans sa dimension totale. 
La mise en lumière de l'inconscient par la psychanalyse leur permit de tirer des conclusions pour l'art. Le réel vécu n'est pas banal à condition de tenir compte de ce qui le sous-tend et le gouverne, une réalité non pas plus haute mais réellement puissante. Il n'y a pas d'incohérence dans l'anarchie du vécu puisque le hasard lui-même n'est que l'aboutissement et l'expression de volontés, de désirs dispersés, incompréhensibles pour une rationalité linéaire mais non moins objective. Mais c'était se situer hors du temps et de l'espace, d'où le besoin ressenti par nombre de surréalistes d'un engagement politique concret.

Cet engagement politique, d'autres artistes en font la pierre angulaire de toute œuvre. Ils épuisent le vécu dans l'instant certes, mais pour comprendre le réel, il importe pour eux de se situer à distance dans une durée. La continuité n'est plus individuelle mais humaine; elle n'est plus personnelle mais collective. Ainsi chaque acte, fût-il un acte de création artistique, se situe dans une durée. C'est l'histoire. Elle détermine non seulement le déroulement des événements collectifs mais chacun de nos actes individuels et les œuvres de création s'incarnent dans une histoire prédéterminée, quelles que soient la volonté ou la perception de l'artiste. Son cuvre appartient aussi à un espace puisque l'histoire se déploie dans des groupes humains concrets, des collectivités, des masses. Aussi l'artiste est-il l'expression d'un groupe. Souhaite-t-il, entend-il lucidement n'être le porte-parole d'aucun groupe, d'aucun peuple, qu'il le serait négativement. Son refus ne met pas en question le concret de l'espace et du temps qui le détermine, mais exprime une attitude personnelle qui dans le refus ne met en lumière que son négativisme. La négation du déterminisme est illusoire. Alors, autant en prendre conscience. L'art est utile, et le fait qu'il serve de décor et d'embellissement n'entame en rien cette assertion. L'artiste qui ne se ferme pas les yeux devant le réel aveuglant, qui ne fuit pas devant le concret, plonge dans l'action, et l'art n'est alors qu'un instrument, et plus l'artiste en est conscient, plus il aiguise son talent dans ce but, plus il est efficace.

Pour Ernst Bloch, l'imaginaire lui-même peut être marqué du sceau de l'efficacité. Aussi, l'utopie ne serait qu'un 
projet. Celui-ci ne se limiterait pas au changement social et économique. Il les comprendrait bien sûr, mais il s'agit d'une modification des rapports humains, d'une révolution dans le goût et dans la sensibilité. La pierre d'angle demeurerait la révolution sociale et économique et l'artiste offre par son projet utopique un programme d'action à l'homme politique.

Refaire le monde peut représenter pour l'artiste une autre forme de choix : abandonner la tentative de le comprendre et à plus forte raison de l'expliquer. Son rapport avec le réel ne serait modifié qu'en apparence. Sacrifier une dimension de son entreprise afin de la rendre efficace n'est finalement qu'une forme d'évasion, même s'il s'agit d'une fuite en avant, d'une incursion dans le futur. Et l'artiste retrouve son angoisse première et son impulsion primordiale. Aucun changement social, aucune révolution politique ne peut régler son rapport avec le réel. Il continuera à produire, quitte à subir l'accusation des nouveaux maitres politiques d'avoir trahi sa mission, d'avoir faussé chemin à ses compagnons de route.

Et le produit est là. Il n'appartient pas à celui qui l'a mis au monde, il n'appartient plus à celui qui l'offre à un public. L'œuvre d'art acquiert dès sa naissance, par rapport à son créateur, une autonomie. Elle a sa vie propre. Elle n'est point indépendance cependant, car ce serait, nous l'avons dit, la vouer à la futilité.

$\mathrm{Si}$, pour nombre d'artistes, l'œuvre est une victoire sur le temps, une affirmation de la vie dans une inscription, dans une durée, cette victoire, éphémère, illusoire, se mue en rêve d'éternité. L'œuvre perpétue le passage de l'artiste en mémoire et assure ainsi sa survie. Ce rêve dont l'artiste ne constate point la confirmation dans le vécu, est l'apanage du public. Le lecteur d'un roman dispose d'une liberté que ne limitent ni temps ni espace. Il redonne vie, par une lecture, à des œuvres contemporaines mais tout autant à celles qui furent produites en d'autres temps et d'autres lieux. L'œuvre retrouve son actualité sous le regard d'un spectateur, même si pour d'autres elle est morte et enterrée. Elle renaît dans une autonomie totale par rapport aux circonstances qui l'ont vu naître, indépendamment de son auteur. Elle est modifiée, changée, 
refaite par le lecteur qui participe ainsi à sa recréation. Elle existe dans un rapport renouvelé par rapport au lecteur. Certes, des œuvres oubliées, enterrées dans des musées ou des bibliothèques, peuvent garder intacte, des siècles durant, leur vie, immobile, inerte, potentielle. Le lecteur suit un itinéraire semblable, même quand il est parallèle, à celui de l'artiste. Par la recréation d'une œuvre, il cherche à donner une cohérence à un monde anarchique, à établir un rapport avec un réel à la fois célébré et masqué par le vécu. Il dépasse ainsi le quotidien en dotant l'instant d'un sens qui en redouble la mesure et qui lui assure une continuité en dépit de l'éphémère. Il conjure ainsi l'insignifiant qui l'assaille, et l'absence qui le menace. Le réel se prolonge dans une représentation qui peut se transformer en réel de remplacement, mais qui peut tout aussi bien donner la mesure du réel, et, dans la distance et le détachement, permettre l'intégration de l'intensité intermittente et discontinue du vécu. Le lecteur s'inscrit ainsi dans une durée. Par les œuvres qu'il fait surgir à volonté à la vie, il se transforme en maitre du temps. Les civilisations sont à sa portée, non comme instrument pour embellir une existence monotone ou le distraire de l'ennui d'un quotidien morne, mais pour doter d'un poids chaque instant, pour octroyer un sens aux gestes du vécu. Ses réactions au monde prolongent celles des hommes. Il n'y a plus de passé, puisque l'œuvre la plus ancienne se transforme par le regard actuel en présence. Et ce regard enlève à la mort sa pesanteur. La vie se perpétue dans le temps et l'artiste inscrit la mémoire des hommes dans une œuvre qui appartient par sa vie, par son dynamisme propre, à l'humanité.

Pour le lecteur, l'œuvre est aussi une révélation d'espace, elle est espace. Le vécu s'épuise dans l'instant mais se déroule dans un lieu. L'espace se transforme en événement. L'œuvre artistique donne à cet événement qui se dissout dans l'insconscient, qui s'épuise dans son propre déroulement, un prolongement, un sens qui ne le dépasse pas mais qui révèle sa dimension. Ainsi l'homme, lecteur et spectateur, qui recrée l'œuvre d'art en fait un événement qui se déroule dans un lieu. Le créateur inscrit l'événement dans un espace. Mais l'espace du vécu n'est pas uniquement une matière, un contenu. Il est 
une dimension de l'espace artistique qui le transforme, le prolonge, le perpétue. L'événement est aussi le lien avec l'autre, et avec les autres, que le lien soit d'amour ou d'échange, de travail ou de jeu. À partir de ce lien, la société consomme les œuvres, les privilégie, les réprime, leur insuffle vie ou les assassine. Les ouvres renaissent comme se transforment et renaissent les liens d'amour et de travail. Ces liens sont explicités, précisés, saisis par la représentation qu'en donnent les ceuvres artistiques. Au premier degré, l'artiste permet au public de regarder le monde qui l'entoure, rend conscient ce regard. L'œuvre est conscience d'un monde. À un autre niveau toute société, aussi éloignée qu'elle apparaisse, est espace du moment qu'un regard d'artiste rend possible le regard du spectateur. C'est le regard lui-même qui crée l'espace, le recrée, le façonne. Un Américain d'aujourd'hui voit sa vie reflétée dans les cuvres d'un Updike ou d'un Bellow, mais à un autre niveau il peut reconnaitre et se reconnaître dans les personnages de Tchekhov et de Balzac.

Chaque œuvre est nouvelle mais elle est aussi un chaînon dans une succession d'œuvres qui l'ont précédée ou qui lui sont contemporaines, sauf qu'elles sont nées dans un autre espace. Ainsi en va-t-il de l'événement. Nos gestes, nos choix font que notre existence soit liée à celle des autres. Nous faisons partie d'une société dont les événements se déroulent à des niveaux différents mais qui, dans le temps, tracent une chronologie. C'est l'histoire, et il y a aussi une histoire de l'art et de la littérature, parallèle à l'histoire tout court.

L'Histoire telle que récitée par les œuvres artistiques est celle du vécu quotidien, revue et remise dans une cohérence qui en fait des civilisations et, au-delà des chronologies, des mythes.

Le public existe dès qu'un individu se retrouve avec d'autres à un spectacle ou dans la lecture d'un roman. La vie personnelle s'étend, échappe à l'individu pour se prolonger et se projeter dans celles d'autres individus. Une tribu est ainsi formée, cellule primaire d'une société à côté de la cellule familiale dont la raison d'être est la continuité de l'espèce par la progéniture. La civilisation de l'écrit, du journal et de la 
transmission des cuvres élargit la tribu, la transforme en nation. L'âge de l'électricité, de la transmission simultanée et universelle des æuvres abat les frontières. Les nations culturelles cèdent le pas devant un public anonyme de consommateurs. Afin qu'il serve les besoins des producteurs industriels de la culture, ce public est réduit à un dénominateur commun. On lui sert des produits interchangeables, préparés au bénéfice d'un public anonyme. L'on tombe dans l'insignifiant et c'est alors que la cellule tribale retrouve une raison d'être, les cultures oubliées renaissent, revendiquent le droit à l'existence, poussant des cris de violence pour être entendues. L'œuvre d'art devient ainsi le définisseur d'une civilisation qu'engloutissent de puissants moyens de communication. La culture est à nouveau interrogation, et l'œuvre d'art cherche la signification au-delà des clichés envahissants.

L'œuvre existe dans l'autonomie mais sa liberté n'est acquise que dans une relation mouvante entre l'artiste et un public. L'œuvre est créée et constamment recréée et c'est dans ce mouvement qu'elle constitue un corps vivant et que l'on peut chercher à en définir la nature.

Les méthodes d'analyse et d'approche de l'œuvre sont, par la force des choses, réductrices. Vue de l'extérieur, l'œuvre ne se laisse pas saisir dans sa totalité. Corps vivant, disions-nous, qui existe dans ses rapports à un public, qui échappe à l'immobilité. Northrop Frye conçoit le rôle du critique comme étant semblable à celui de l'écrivain. La matière première de l'écrivain est le vécu et celle du critique est l'œuvre de l'écrivain. Ce dernier est comme le chirurgien qui dissèque un corps, qui en révèle les structures. En cela l'œuvre appartient à un domaine plus vaste, celui de l'esprit qui englobe et la religion et l'inconscient. Frye décèle le déroulement mythologique de l'esprit humain dans l'œuvre littéraire. Cette méthode riche, pénétrante, éclairante, présente néanmoins de graves difficultés et comprend de sérieuses lacunes. L'œuvre est réduite à un corps que l'on dissèque. Il est par conséquent mort. Ainsi une dimension essentielle échappe à cette analyse. Et si l'on se penche sur les mystères de la structure, l'on néglige forcément d'établir une hiérarchie qualitative. Cette hiérarchie entre œuvres majeures et mineures, cuvres qui font 
partie de la mémoire de l'humanité et œuvres justement vouées à l'oubli, chaque méthode d'analyse cherche à l'établir. Mais il s'agit d'une hiérarchie qui privilégie une dimension de l'œuvre, celle qui répond à ses critères, et qui sacrifie les autres, qui passe à côté de l'ensemble, du corps vivant.

Cette méthode de dissection est poussée à l'extrême par la linguistique et la sémiotique. En reliant l'œuvre à une mythologie, l'anatomie critique de Frye fait appel à une lumière braquée de l'extérieur sur le corps de l'œuvre. À la limite, elle peut être récusée comme étant étrangère à l'œuvre.

Les linguistes et les sémioticiens pénètrent l'œuvre, la scrutent de l'intérieur. Ils lui reconnaissent une entière autonomie. Elle est texte et l'auteur, l'artiste, n'est qu'un instrument par lequel le texte émerge à la surface. Émerge d'où? Peu importe. Il exprime une structure qu'il contient dans sa totalité. C'est donc à l'intérieur du texte que l'on doit chercher le secret, le mystère. Si l'auteur est un instrument quasi anonyme, le public est absent. On cherche ainsi à atteindre à une grammaire, à des règles précises de la création. Démarche qui serait calquée sur celle de la recherche scientifique. Et l'on a recours à la plus abstraite des sciences, les mathématiques, pour atteindre l'objectivité. Encore là, la qualité de l'œuvre est secondáire puisqu'il s'agit du goût, donc de subjectivité. À la limite, toutes les cuvres, les plus médiocres comme les plus achevées, s'équivalent. Et l'on passe plus de temps à saisir les mystères des structures d'un texte qu'à le lire. Greimas dit clairement qu'il ne fait point de critique. À la limite, la littérature n'est pour lui qu'un prétexte pour développer une science sémiotique. L'analyse de la structure d'une œuvre peut s'effectuer parallelement à l'œuvre, indépendamment de sa dimension réelle. C'est une tautologie. Comme la philologie et la grammaire ne donnent d'un texte qu'une description des composantes linguistiques.

Toute aussi partielle est la méthode psychanalytique. Freud réduit la création artistique à un phénomène pathologique. Même si c'était vrai, on serait à côté de la question. Que l'artiste soit jeune ou vieux, grand ou petit, malade ou en bonne santé, son rapport avec le public est le produit qu'il 
offre et c'est le produit qui compte et qu'il importe de saisir, quelle que soit la motivation de l'artiste qui l'a mis au monde.

La méthode historique se contente d'un déroulement dans le temps sans tenir compte de ce qu'une cuvre ancienne, définie dans un temps et un espace, y échappe constamment par une présence dans une conscience contemporaine qui en fait une ceuvre d'actualité.

La sociologie ne retient qu'un aspect de l'œuvre. Et l'idéologie, quelle qu'elle soit, cherche à exploiter l'œuvre, à exiger qu'elle soit utile et pertinente. Encore là l'œuvre lui échappe, même si elle cherche à l'emprisonner.

Dans son livre sur la nouvelle critique, Serge Doubrovsky préconise la méthode philosophique comme éclairage de l'œuvre littéraire. Je vois là aussi une limite. Les concepts peuvent représenter un carcan. On peut les déceler à partir des œuvres. Ils ne peuvent les conditionner. Pour mettre de l'ordre dans l'anarchie du réel, l'artiste cherche une cohérence qui ne serait pas forcément logique ou rationnelle. À moins d'accepter que l'œuvre d'art ait sa propre logique et sa rationalité particulière.

Un faisceau d'éclairages divers, linguistiques, philosophiques, sociologiques et psychologiques, permettrait une vision de l'ceuvre d'art dans l'immensité et la fragilité de son autonomie. Cet éclairage détaché, s'il donne la mesure de l'œuvre, demeure néanmoins à l'extérieur.

Seule une cuvre d'art permet une connaissance réelle d'une autre ouvre d'art. Un texte littéraire nie ceux qui l'ont précédé, et en les niant les confirme. En s'affirmant comme corps vivant, il affirme la vie de ce qu'il nie. Il suit en cela le mouvement même de la vie, qui échappe à toute analyse en épousant le surgissement des signes d'animation dans l'ombre même de la mort. 